\title{
GAMIFICACIÓN: LA VUELTA AL MUNDO EN 80 DÍAS
}

Gamification: Around the world in eighty days

Gamificación: Volta ao Mundo em Oitenta Dias

Tania Ordiz Carpintero

CEIP Compostilla, León, España. Teléfono: +34 645930916. Correo electrónico: carpiny82@hotmail.com

\section{Resumen}

La experiencia muestra una aplicación de Gamificación en un centro de educación primaria. En ella se intenta unir el juego con el aprendizaje, con la intención de buscar una mayor motivación por parte de los alumnos a través de experiencias positivas y una participación activa vinculándola a diferentes aplicaciones como ClassDojo, Kahoot o Plickers. La Gamificación supone una alternativa pedagógica al involucrar al alumnado, a través del juego de retos, en su propio proceso de aprendizaje. La experiencia muestra que la propuesta incentiva el ánimo de superación del alumnado, consiguiendo una mayor interiorización de conocimientos, actitudes, habilidades y destrezas.

Palabras clave: Gamificación, motivación, juego, participación activa

\begin{abstract}
The experience shows to apply of Gamification in a primary school. It tries to join the game with learning in order to look for a better motivation on the part of students through positive experiences and active participation linking it to different applications like ClassDojo, Kahoot or Plickers. Gamification is a pedagogical alternative to involve students, through the game of challenges, in their own learning process. The experience shows that the proposal encourages students to improve their own learning process, getting a better internalization of knowledge, attitudes, skills and abilities.
\end{abstract}

Keywords: Gamificatión, motivación, play, active participation

\section{Resumo}

A experiência mostra uma aplicação de Gamification em educação primária. Nele você tentar entrar no jogo com a aprendizagem, com a intenção de alcançar uma maior 
motivação dos alunos através de experiências positivas e sua participação ativa, ligandoo a diferentes aplicações, tais como ClassDojo, Kahoot ou Plickers. O Gamification representa uma alternativa educacional para envolver os alunos, através do jogo de desafios em seu próprio processo de aprendizagem. A experiência mostra que a proposta incentiva o animo de superação do aluno e atingindo uma maior interiorização de conhecimentos, atitudes e habilidades.

Palavras-chave: Gamificação, motivação, jogo, participação

\title{
1. Introducción
}

Las tendencias pedagógicas actuales abogan, de una u otra forma, por un desarrollo del proceso de enseñanza-aprendizaje que dote de un protagonismo significativo al alumno sobre su propio aprendizaje (Aretio, 2016; Carpena, Cataldi \& Muñiz, 2012).

La Gamificación es una técnica que parte del mundo empresarial, y que se ha llevado al mundo educativo por los excelentes resultados. En este sentido, Martín y Hierro (2013) entienden la gamificación como:

\begin{abstract}
una técnica, un método y una estrategia a la vez. Parte del conocimiento de los elementos que hacen atractivos a los juegos e identifica, dentro de una actividad, tarea o mensaje determinado, en un entorno de NO-juego, aquellos aspectos susceptibles de ser convertidos en juego o dinámicas lúdicas. Todo ello para conseguir una vinculación especial con los usuarios, incentivar un cambio de comportamiento o transmitir un mensaje o contenido. Es decir, crear una experiencia significativa y motivadora (Martín \& Hierro, 2013, p. 15).
\end{abstract}

La intención de esta comunicación es presentar una experiencia de Gamificación a través de un juego de retos, como núcleo vertebrador del proceso de aprendizaje, e incorporando aplicaciones (ClassDojo, Kahoot, Plickers o Croma) para fomentar la implicación del alumnado, comprobar su aprendizaje, aumentar su responsabilidad y mejorar sus comportamientos.

\section{Contextualización}

La experiencia se lleva a cabo en el CEIP de Compostilla (Ponferrada, León). En concreto se realizó con un grupo de $5^{\circ}$ de Primaria, compuesto por 18 alumnos. La duración de la experiencia abarcó todo el segundo trimestre y parte del tercero.

\section{Diseño y desarrollo}

Durante el primer trimestre, los alumnos trabajaron estrategias cooperativas como el folio giratorio (Pujolas, 2008), el puzle de Aronson (Aronson, 1978) o cabezas viajeras Gamificación: La vuelta al mundo en 80 días 
unidas (Kagan, 1994). Sin embargo, éstas no generaron ni la implicación ni el aprendizaje esperado, manteniéndose falta motivación hacia el aprendizaje, de responsabilidad hacia sus tareas, así como de hábitos de estudios.

Por ello, a partir de todos estos resultados se diseñó la actividad: "La vuelta al mundo en 80 días”; un juego de retos con una duración de 80 días escolares y en la que los alumnos fueron visitando los diferentes continentes (figura 1).

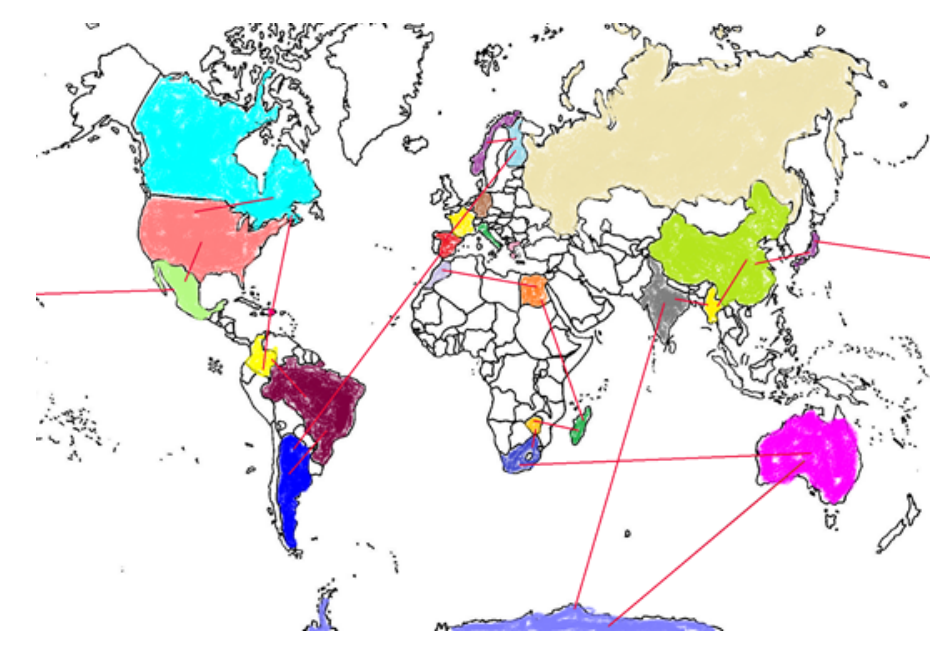

Figura 1.

Recorrido realizado en la gamificación

El proceso de aplicación se organizó en las siguientes fases:

Fase 1: El juego comenzó proyectando un vídeo sobre viajes, el cual despertó la curiosidad del alumnado y, posteriormente, se dieron a conocer las reglas del juego.

Simultáneamente se presentó la aplicación ClassDojo como recurso motivador al identificar los logros individuales y grupales en el viaje, proporcionando el control de los puntos del juego. Para ello, los alumnos crearon sus propios avatares como se observa en la figura 2.

Se establecieron 6 niveles, partiendo del nivel "rutero" e intentando llegar al nivel máximo “aventurero” (Figura 3). Para pasar de nivel debían conseguir cien puntos. Estos puntos se consiguen con diferentes retos: estudiar todos los días, hacer los deberes, la nota del examen o realizando retos personales (Figura 4). Éstos últimos consisten en pedir a cada alumno aquello en lo que, tiene dificultad o un hábito que requiere cambiar como: preparar solo la mochila, organizar su pupitre, etc.). 


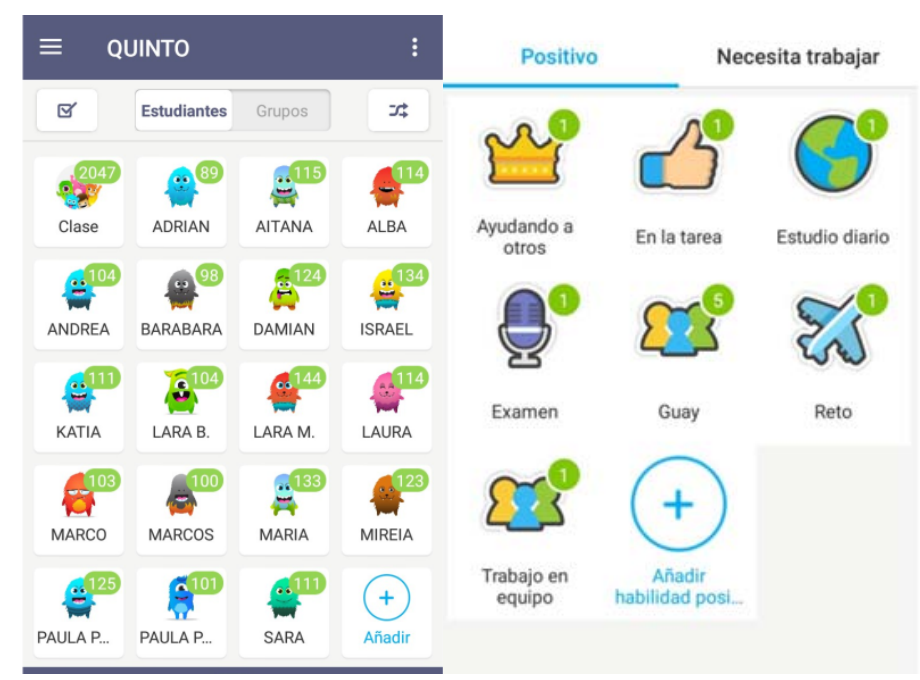

Figura2.

ClassDojo del aula.

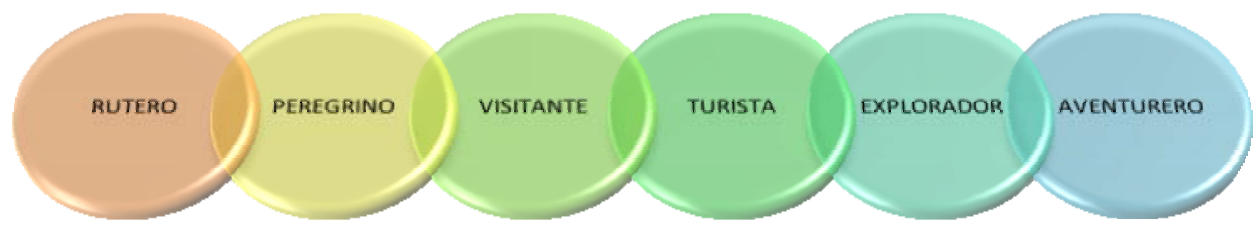

Figura 3.

Recorrido realizado en el juego de retos.

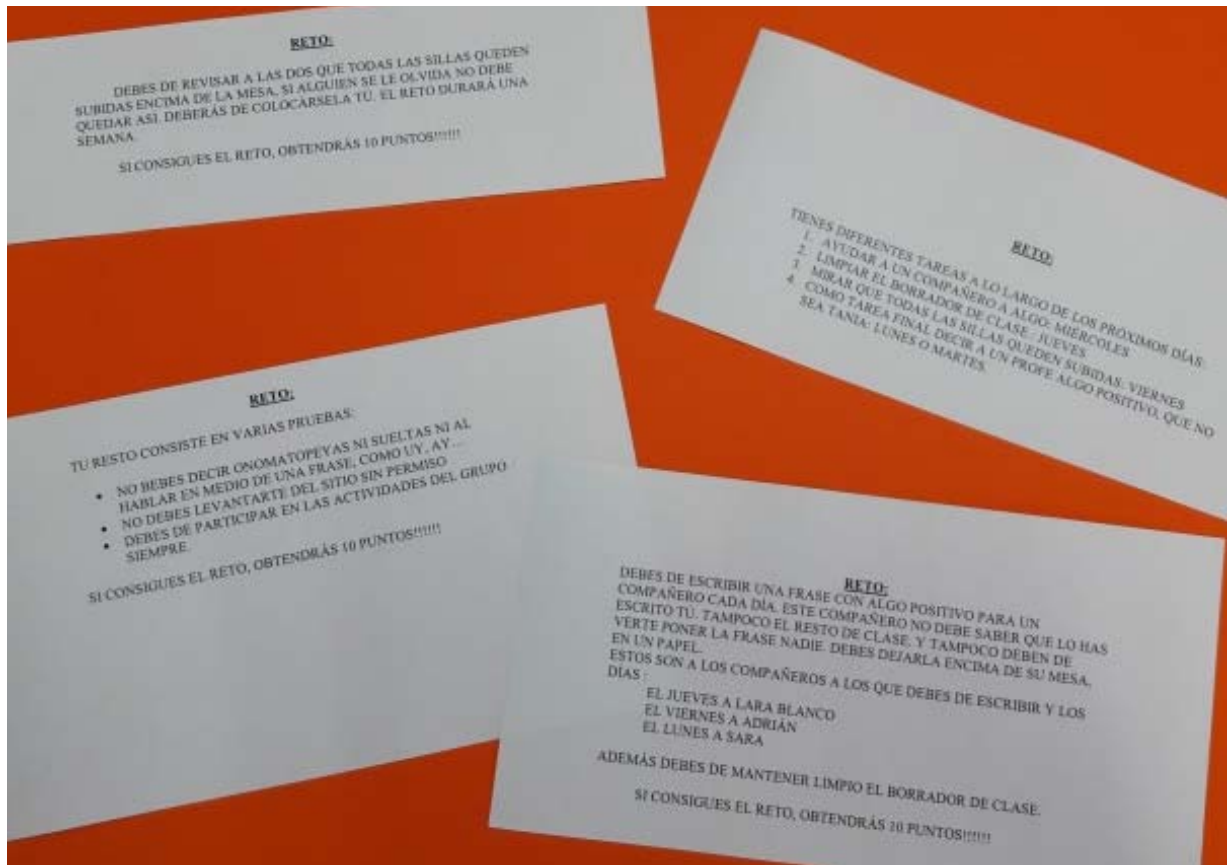

Figura 4.

Ejemplos de retos individuales para conseguir puntos

Gamificación: La vuelta al mundo en 80 días 
Fase 2: Iniciado el juego, a lo largo de los 80 días se fueron visitando diferentes países, dedicando a cada uno varios días para ver videos del país, curiosidades, fotos, dinero si es diferente al nuestro, ropa, objetos típicos, etc. Para ello, se crearon pasaportes (Figura 5) que permitían anotar los resultados; éstos se iban sellando a la entrada y a la salida de cada país a modo de registro de actividad lograda.

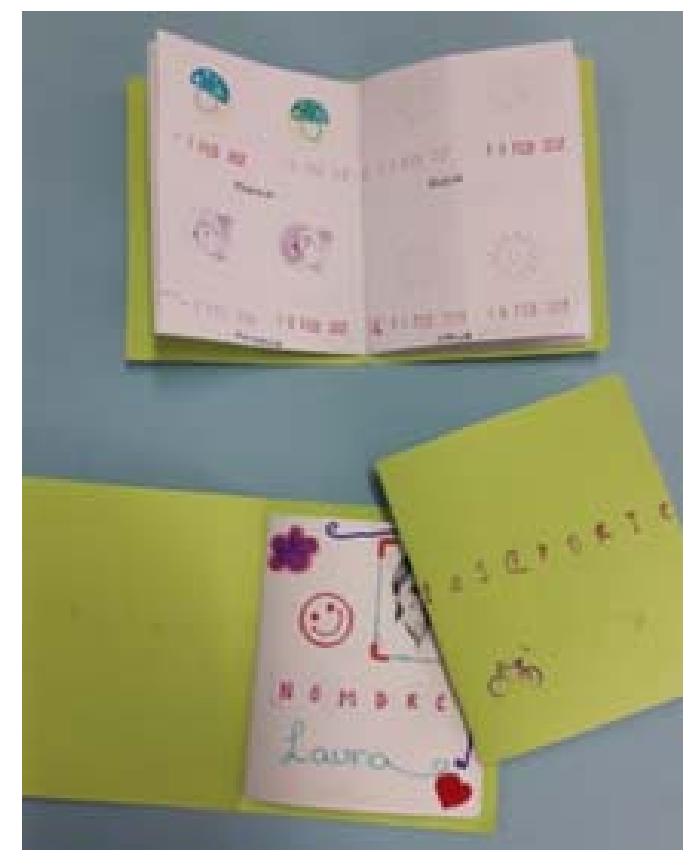

Figura 5

Pasaportes

Fase 3: Al finalizar la estancia en cada continente después de visitar el número de países previamente decididos, se realizaba una prueba de comprobación de conocimientos para poder cambiar de continente y continuar jugando. Estas pruebas fueron muy diferentes, por ejemplo: caza tesoros, pruebas de orientación, pruebas de pistas, para las cuales se utilizaron diferentes medios como, por ejemplo, códigos QR, donde se mandaba a los grupos a diferentes lugares donde tenían que responder diferentes preguntas, etc. En éstas pruebas, las preguntas y la temática versaban sobre los aspectos relacionados con los países visitados o con los contenidos curriculares vistos temporalmente a la par de ese continente. Las puntuaciones de cada una dependían de la cantidad de preguntas, de la dificultad...

Para poder viajar de un continente a otro, los alumnos necesitaban un medio de transporte, aspecto que generaba tener que realizar una prueba grupal. Ésta consistía en resolver de forma positiva una prueba de consolidación de contenidos. Para la 
realización de ésta se utilizaron aplicaciones como Plickers o Kahoot. La prueba además nos servía para repasar antes del examen los contenidos trabajados en las diferentes áreas.

Finalmente, para terminar el viaje, los alumnos recibieron un reconocimiento individual, en forma de banda (Figura 6), la cual contenía todas las medallas obtenidas en cada uno de los niveles, y un reconocimiento grupal, que consistía en una salida al entorno cercano.

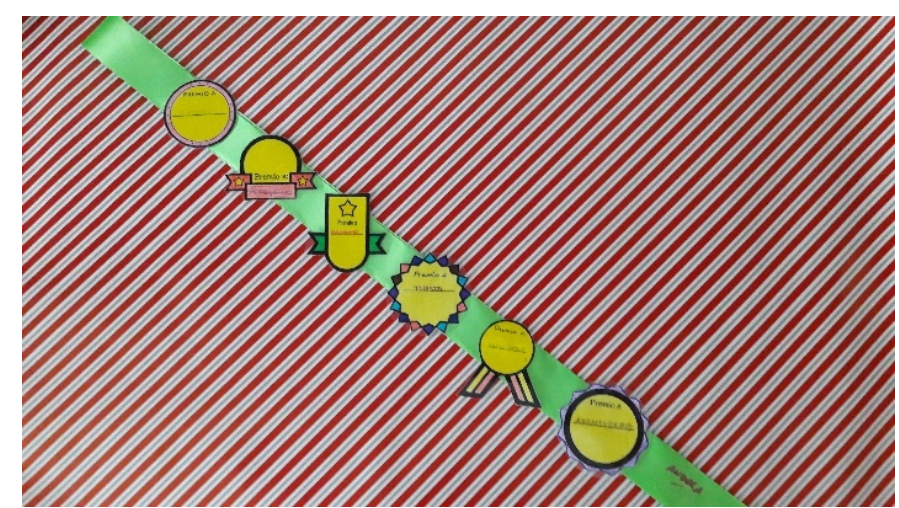

\section{Figura 6}

Banda de una alumna con las medallas conseguidas

La experiencia de Gamificación finaliza cuando los alumnos sellan todo su pasaporte y realizan un álbum de recuerdos con las fotos de los lugares visitados a través de la técnica del Croma.

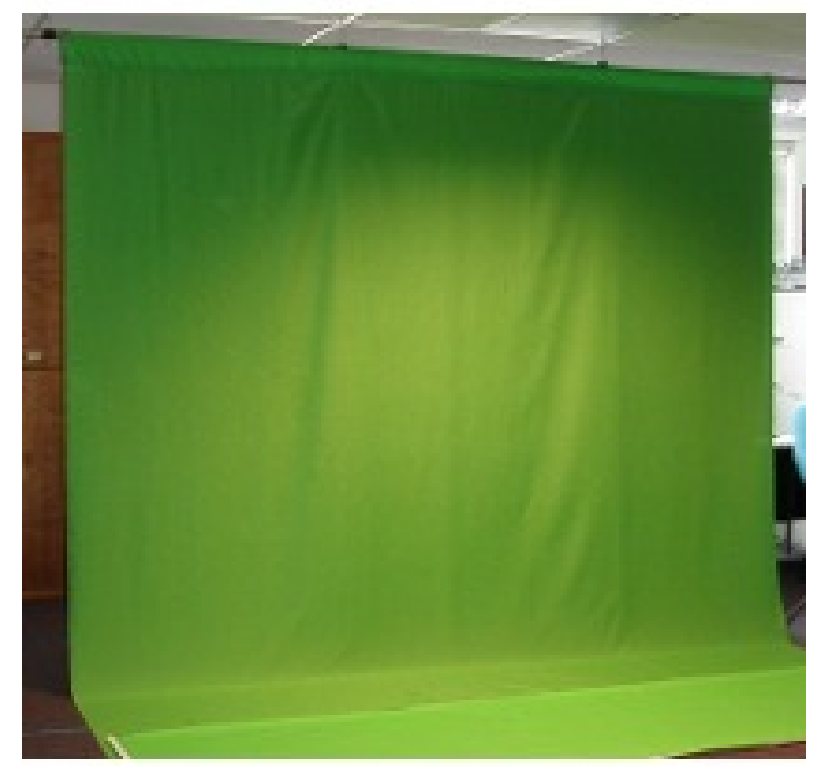

Figura 7

Técnica del croma

Gamificación: La vuelta al mundo en 80 días 


\section{Evaluación y conclusiones}

Respecto a la evaluación de la experiencia, se han utilización listas de control, registros de anécdotas, las aplicaciones digitales como ClassDojo para el registro de actividad o Kahoot y Plickers para la comprobación de aprendizaje, así como otras pruebas específicas. Sin embargo, los cuestionarios de satisfacción a los alumnos y las entrevistas a las familias han permitido constatar que la experiencia ha sido muy positiva.

En conclusión, tras los malos resultados del primer trimestre, la aplicación en el segundo y tercero ha permitido comprobar cómo se han mejorado la implicación de los alumnos, su comportamiento y su responsabilidad individual y grupal hacia el aprendizaje.

\section{Referencias}

Aretio, L. G. (2016). El juego y otros principios pedagógicos. Su pervivencia en la educación a distancia y virtual. RIED. Revista Iberoamericana de Educación a Distancia, 19(2), 9-23. Recuperado de http://revistas.uned.es/index.php/ried/article/view/16175

Aronson, W. (1978). The jigsaw classroom. Beverly Hills, CA: Sage publications

Carpena, N., Cataldi, M., \& Muñiz, G. (2012). En busca de nuevas metodologías y herramientas aplicables a la educación. Repensando nuestro rol docente en las aulas.

Recuperado de .http://papers.cumincad.org/data/works/att/sigradi2012_85.content.pdf

Kagan, S. (1994). Cooperative learning. San Clemente, CA: Resources for Teachers.

Kapp, K. (2012). The Gamification of Learning and Instruction: Game-Based Methods and Strategies for Training and Education. San Francisco: John Wiley \& Sons.

Marín, I., \& Hierro E. (2013). El poder del juego en la gestión empresarial y la conexión con los clientes. Barcelona: Urano / Empresa activa.

Pujolàs, P. (2008). 9 ideas clave: El aprendizaje cooperativo. Barcelona: Graó. 